Portland State University

PDXScholar

Computer Science Faculty Publications and

Presentations

Computer Science

2013

\title{
Interpreting Individual Classifications of Hierarchical Networks
}

Will Landecker

Portland State University, will.landecker@gmail.com

Michael David Thomure

Portland State University, mthomure@gmail.com

Luis M.A. Bettencourt

Santa Fe Institute

Melanie Mitchell

Portland State University, mm@pdx.edu

Garrett T. Kenyon

Los Alamos National Laboratory

See next page for additional authors

Follow this and additional works at: https://pdxscholar.library.pdx.edu/compsci_fac

Part of the Artificial Intelligence and Robotics Commons, and the Theory and Algorithms Commons Let us know how access to this document benefits you.

\section{Citation Details}

Landecker, Will; Thomure, Michael David; Bettencourt, Luis M.A.; Mitchell, Melanie; Kenyon, Garrett T.; and Brumby, Steven P., "Interpreting Individual Classifications of Hierarchical Networks" (2013). Computer Science Faculty Publications and Presentations. 165.

https://pdxscholar.library.pdx.edu/compsci_fac/165

This Working Paper is brought to you for free and open access. It has been accepted for inclusion in Computer Science Faculty Publications and Presentations by an authorized administrator of PDXScholar. Please contact us if we can make this document more accessible: pdxscholar@pdx.edu. 


\section{Authors}

Will Landecker, Michael David Thomure, Luis M.A. Bettencourt, Melanie Mitchell, Garrett T. Kenyon, and Steven P. Brumby 


\section{Interpreting Individual Classifications of Hierarchical Networks}

Will Landecker

Michael D. Thomure

Luís M. A. Bettencourt

Melanie Mitchell

Garrett T. Kenyon

SFI WORKING PAPER: 2013-02-007

SFI Working Papers contain accounts of scientific work of the author(s) and do not necessarily represent the views of the Santa Fe Institute. We accept papers intended for publication in peer-reviewed journals or proceedings volumes, but not papers that have already appeared in print. Except for papers by our external faculty, papers must be based on work done at SFI, inspired by an invited visit to or collaboration at SFI, or funded by an SFI grant.

(CNOTICE: This working paper is included by permission of the contributing author(s) as a means to ensure timely distribution of the scholarly and technical work on a non-commercial basis. Copyright and all rights therein are maintained by the author(s). It is understood that all persons copying this information will adhere to the terms and constraints invoked by each author's copyright. These works may be reposted only with the explicit permission of the copyright holder.

www.santafe.edu 


\title{
Interpreting Individual Classifications of Hierarchical Networks
}

\author{
Will Landecker*, Michael D. Thomure*, Luís M. A. Bettencourt ${ }^{\dagger \ddagger}$, \\ Melanie Mitchell ${ }^{* \dagger}$, Garrett T. Kenyon ${ }^{\ddagger}$, and Steven P. Brumby ${ }^{\ddagger}$ \\ *Department of Computer Science \\ Portland State University \\ \{landeckw, thomure, mm @ cs.pdx.edu \\ †Santa Fe Institute \\ bettencourtasantafe.edu \\ $\ddagger$ Los Alamos National Laboratory \\ \{gkenyon, brumby\}@lanl.gov
}

\begin{abstract}
Hierarchical networks are known to achieve high classification accuracy on difficult machine-learning tasks. For many applications, a clear explanation of why the data was classified a certain way is just as important as the classification itself. However, the complexity of hierarchical networks makes them ill-suited for existing explanation methods. We propose a new method, contribution propagation, that gives per-instance explanations of a trained network's classifications. We give theoretical foundations for the proposed method, and evaluate its correctness empirically. Finally, we use the resulting explanations to reveal unexpected behavior of networks that achieve high accuracy on visual object-recognition tasks using well-known data sets.
\end{abstract}

\section{INTRODUCTION}

In machine learning, a model is learned from structure in the data. When using machine-learning techniques, one hopes that the learned structure generalizes well to yet-unseen data. However, many machine-learning techniques result in blackbox models, making it difficult to understand the nature of the learned structure. With interpretable methods that explain the interactions between data and model, we can ensure that our learned models have learned relevant structure from the data, rather than spurious statistics.

This paper focuses on a family of hierarchical networks in which the operations computed at each layer alternate between some type of pattern matching (e.g., convolution, radial basis function) and subsampling (e.g., mean, maximum). Such networks have been used extensively in the machine learning community, and are known to achieve high classification accuracy on a variety of tasks such as phoneme recognition [1], text document classification [2], and visual object recognition [3]. Due to their complexity, such networks are often treated as black boxes, giving accurate solutions to difficult problems without explaining how their solutions were found.

However, for hierarchical networks to be used for applications in which each classification carries high risk (e.g., medical diagnosis or financial decisions), it may be necessary for these networks to explain the evidence for each classification. More generally, for any classification method, it is important

(C) 2013 IEEE Symposium Series on Computational Intelligence to verify that the network's results are due not to anomalies or accidental correlations in particular data sets, but to features of the data that make sense for the general task at hand.

Given an instance $\mathbf{x}=\left(x_{1}, x_{2}, \ldots, x_{n}\right)$ classified as $\hat{y}(\mathbf{x}) \in$ $\{1,-1\}$, we seek a method to explain the relative importance of each input $x_{i}$ to the instance's classification. Poulin et al. [4] offer such an explanation method for the case of additive classifiers. For an additive classifier $\hat{y}(\mathbf{x})=\operatorname{sgn}\left[\sum_{i=1}^{n} f_{i}\left(x_{i}\right)+b\right]$, Poulin et al. define the contribution of feature $x_{i}$ to be $f_{i}\left(x_{i}\right)$. However, the classifications of hierarchical networks cannot be explained with this method: even if the features calculated by the network are classified by an additive classifier, the highlevel features themselves are generally nonadditive functions of the network's low-level inputs.

We address this limitation with a new method called contribution propagation. In short, we calculate contributions of high-level features as in Poulin et al. [4], and then work backward from the features to the network's input, determining the relative contribution of each node to its parent nodes. This process gives us a well-founded explanation for individual classifications: given a single classified datum, our method explains how important each part of the datum (i.e., each entry in the input vector) was to that classification.

In what follows, we review the literature related to this research, and in Section II, we introduce notation and review the architecture of hierarchical networks. Section III gives the details of the contribution-propagation method. Section IV discusses the implementation details of our network, and we give experimental results in Section V. We conclude with directions for further research and open questions.

\section{A. Related Work}

Rule extraction refers to a family of methods that explain the general rules used by a network by analyzing the network's internal weights [5]. Similarly, one can visualize the network topology in order to understand the overall strategies that might be used [6]. The extracted rules are not specific to the model's decision for any particular datum. Rather, they describe the general behavior of the model over a large set

To appear in Computational Intelligence and Data Mining (CIDM) 2013 
of data. Thus, rule extraction does not provide the type of information we seek: a fine-grained explanation of what caused a single datum's classification.

In an attempt to provide such explanations, recent research has treated the entire classification system as a single black box in order to provide classifier-independent explanations [7]. These methods avoid dealing with the internal calculations of the system at the expense of sampling from an exponentially large set of possible inputs. For classifiers with large input spaces, as is often the case with hierarchical networks, these methods introduce unwanted dependencies on sampling.

The gradient approach is one way to explain individual classifications [8]. Given an instance $\mathbf{x}=\left(x_{1}, x_{2}, \ldots\right)$ and its classification $\hat{y}(\mathbf{x})=\operatorname{sgn}[f(\mathbf{x})]$, this approach defines the importance of feature $x_{i}$ to be $\frac{\partial f}{\partial x_{i}}$. Intuitively, $x_{i}$ is important if $f$ is sensitive to small changes in $x_{i}$. However, the gradient approach fails to tell us how much each feature contributed to the output. Consider the simple example of a linear classifier $f(\mathbf{x})=\mathbf{w}^{\top} \mathbf{x}$, where $\mathbf{w}=(2,2)$. An input $\mathbf{x}=\left(x_{1}, x_{2}\right)=$ $(3,-1)$ gives output

$$
f(\mathbf{x})=2 \cdot 3+2 \cdot(-1)=6-2=4 .
$$

A different input $\mathbf{x}^{\prime}=\left(x_{1}^{\prime}, x_{2}^{\prime}\right)=(-1,3)$ gives output

$$
f\left(\mathbf{x}^{\prime}\right)=2 \cdot(-1)+2 \cdot 3=-2+6=4 .
$$

The gradient approach would tell us that our function $f$ is equally sensitive to all inputs in both cases $\left(\frac{\partial f}{\partial x_{i}}=2\right)$. However, this analysis fails to tell us that in the first case, the positive output of $f$ is due to the first feature $x_{1}$; and in the second case, the positive output of $f$ is due to the second feature $x_{2}^{\prime}$.

The contribution approach of Poulin et al. [4], on the other hand, perfectly captures this information. Using this approach, we would determine that for the first example, the contributions of $x_{1}$ and $x_{2}$ are 6 and -2 , respectively. For the second example, the contributions of $x_{1}^{\prime}$ and $x_{2}^{\prime}$ are -2 and 6 , respectively. Thus contributions (which we explore in more detail below) give us fine-grained explanations of the degree to which each input contributed the output, while the gradient approach loses much useful information about the classification.

\section{PRELIMINARIES}

\section{A. Notation}

We use italicized letters to denote real numbers and bold letters to denote real vectors, such as $\mathbf{x}=\left(x_{1}, \ldots, x_{n}\right) \in \mathbb{R}^{n}$. The L2 norm of a vector, denoted $\|\mathbf{x}\|$, is calculated as $\sqrt{\sum_{i=1}^{n} x_{i}^{2}}$. A node is a real-valued function. Capital letters $X$ refer to the identity of a node, the function calculated by that node, or the value returned by the node's function. The intended use will be clear from context.

For a given hierarchical network, $X_{i}^{\ell}$ refers to the $i^{\text {th }}$ node in the $\ell^{\text {th }}$ layer. The vector $\mathbf{X}^{\ell}=\left(X_{1}^{\ell}, X_{2}^{\ell}, \ldots\right)$ denotes all nodes in layer $\ell$. We write $X^{\ell}$ to mean some node in the $\ell^{\text {th }}$ layer of the network, or merely $X$ to denote any particular node in the network. The children of node $X^{\ell}$, denoted ch $\left(X^{\ell}\right)$, are the set of nodes that are the inputs to node $X^{\ell}$. In a slight abuse of notation, we sometimes treat $c h\left(X^{\ell}\right)$ as a vector; the intended use will be clear by context. Similarly, the parents of node $X^{\ell}$, denoted pa $\left(X^{\ell}\right)$, are the set (or vector) of nodes that receive $X^{\ell}$ as input. In what follows, we will restrict the inputs of any node to come from only the preceding layer. Thus $\mathbf{X}^{\ell-1} \supseteq \operatorname{ch}\left(X^{\ell}\right)$ and $\mathbf{X}^{\ell+1} \supseteq$ pa $\left(X^{\ell}\right)$. A network has $L$ layers $(\ell \in[1, \ldots, L])$. Thus the output of the network, often called the feature vector, is $\mathbf{X}^{L}$. For convenience, the input to the network is denoted simply $\mathbf{x}$. Lastly, let $\mathbf{x} \in \mathbb{R}^{n}$, and for any node $X_{i}^{1}$ in the network's first layer, ch $\left(X_{i}^{1}\right) \subseteq \mathbf{x}$.

\section{B. Hierarchical Networks}

Hierarchical networks comprise a large family of machinelearning models such as HMAX [3], [9], convolutional neural networks [2], and others. In order to make our analysis concrete, in this work we focus on the well-known family of hierarchical networks described by HMAX. However, it should be noted that our contribution propagation method is applicable to the more general class of feed-forward hierarchical networks. We briefly review the architecture of HMAX-like networks here. Details of our implementation are given in the Methods section.

In a trained network, a node $X_{k}^{\ell}$ in an odd-numbered layer computes the radial basis function $(\mathrm{RBF})$

$$
X_{k}^{\ell}=\exp \left(-\beta\left\|\operatorname{ch}\left(X_{k}^{\ell}\right)-\mathbf{P}_{k}^{\ell}\right\|^{2}\right),
$$

where $\mathbf{P}_{k}^{\ell}$ and $\beta$ are parameters of the model. We refer to $\mathbf{P}_{k}^{\ell}$ as the kernel of node $X_{k}^{\ell}$. Following Serre et al. [3], we refer to these layers as $S$ (simple cell) layers, with $S 1$ being the first $S$ layer, and so on. Nodes $X_{h}^{\ell+1}$ in even layers compute a maximum of their inputs:

$$
X_{h}^{\ell+1}=\max _{X_{k}^{\ell} \in \operatorname{ch}\left(X_{h}^{\ell+1}\right)} X_{k}^{\ell}
$$

Again following Serre et al. [3], we refer to these layers as $C$ (complex cell) layers.

Figure 1 shows a hierarchical network with two $S$ layers and two $C$ layers, whose input $\mathbf{x}$ consists of the gray-scale pixel values of an image. The output of the network (i.e., the output of the $C 2$ layer) is the feature vector $\mathbf{X}^{L}$, which is given to a trainable classifier (in this case, a linear support vector machine (SVM)).

\section{METHODS}

Given an instance $\mathbf{x}=\left(x_{1}, x_{2}, \ldots, x_{n}\right)$, its corresponding feature vector $\mathbf{X}^{L}$ (i.e., the final layer of the network), and the feature vector's binary classification $\hat{y}\left(\mathbf{X}^{L}\right)=\operatorname{sgn}\left[f\left(\mathbf{X}^{L}\right)\right] \in$ $\{1,-1\}$, we ask what portion of the value $f\left(\boldsymbol{X}^{L}\right)$ came from input $x_{i}$ ? In this work we assume that the "score" $f\left(\mathbf{X}^{L}\right)$ is an estimate of the classifier's confidence in its classification of $\mathbf{X}^{L}[10]$.

Rather than answer this question for the entire classifier and network as one large black box, our approach is to analyze each node (as well as the classifier) sequentially. Working from the classifier back to the inputs, we determine the contributions of the nodes in layer $L$ to the classifier, then the contributions of the nodes in layer $L-1$ to the nodes in layer $L$, and so on until we have calculated the contribution of the inputs (e.g., the contribution of each pixel in the image of Figure 1). This process, which we call contribution propagation, is ideal for interpreting the classifications made by complex networks 


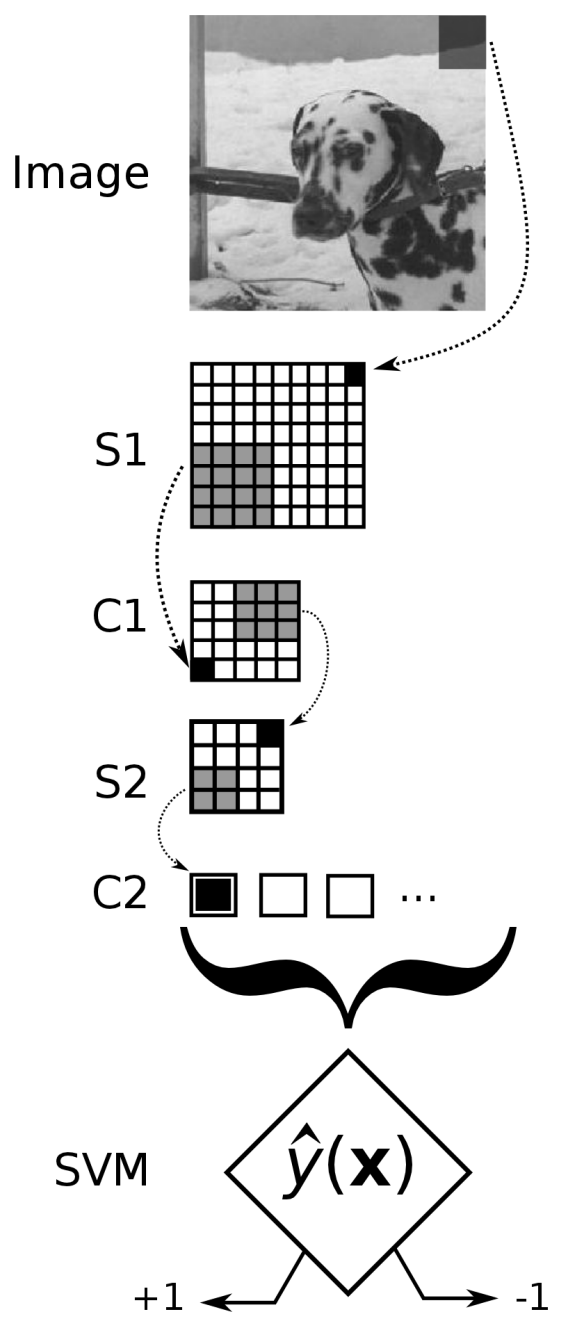

Fig. 1. A hierarchical network taking an image as input. Only a small subset of each layer is shown. Each small square is a node in the network. Computation flows from top (Image) to bottom (SVM). Dashed arrows illustrate the local connectivity of the network: a small subset of each layer (gray group of nodes) is fed as input to a single node (black) in the following layer. The vector consisting of each $C 2$ output is the feature vector used for training and testing the SVM.

which are too large and complicated to interpret directly, yet each individual calculation (i.e., calculating the value output by any single node in the network) is simple.

It is crucial to note that our contribution-propagation algorithm is used only in explaining a model's classification. The classification itself is performed by a trained hierarchical model of the user's choosing. Separation between the classification and explanation algorithms allows our explanation method to be used in a variety of settings with a variety of models. Pseudocode for our algorithm is given in Figure 2.

In the remainder of this section, we describe the contribution-propagation algorithm at a relatively high level. We then discuss how to apply the algorithm to the particular type of hierarchical network discussed in Section II-B. This involves deriving equations specific to the linear SVM, RBF, and maximum operator.

\section{A. The Contribution-Propagation Algorithm}

The purpose of the contribution-propagation algorithm is to provide an interpretable explanation of which components of the input were responsible (and to what degree) for a given datum's classification. When the classification is the result of many simple calculations, as is the case with hierarchical networks, we form an overall description of an input's importance (i.e., this pixel was important to the classification) by analyzing the internal calculations of the network (i.e., node $X_{i}^{\ell}$ was important to node $X_{j}^{\ell+1}$ ).

The central idea of contribution propagation is that a node was important to the classification if it was important to its parents, and its parents were important to the classification. Mathematically,

$$
\mathcal{C}\left(X_{i}^{\ell}\right) \stackrel{\text { def }}{=} \sum_{X_{j}^{\ell+1} \in \operatorname{pa}\left(X_{i}^{\ell}\right)} \mathcal{C}\left(X_{i}^{\ell} \mid X_{j}^{\ell+1}\right) \mathcal{C}\left(X_{j}^{\ell+1}\right) .
$$

In Equation 1, $\mathcal{C}\left(X_{i}^{\ell}\right)$ is the contribution of node $X_{i}^{\ell}$ to the classification; similarly, $\mathcal{C}\left(X_{j}^{\ell+1}\right)$ is the contribution of node $X_{j}^{\ell+1}$ to the classification. Finally, $\mathcal{C}\left(X_{i}^{\ell} \mid X_{j}^{\ell+1}\right)$ is the partial contribution of node $X_{i}^{\ell}$ to its parent node $X_{j}^{\ell+1}$. Informally, this value represents how important $X_{i}^{\ell}$ was to $X_{j}^{\ell+1}$. We will give explicit definitions for these terms in later sections; for the moment, we complete our general discussion of the algorithm.

The contribution-propagation algorithm starts by calculating the contribution $\mathcal{C}\left(X_{i}^{L}\right)$ of each top-level feature $X_{i}^{L}$ to the classifier. The algorithm then iteratively descends through the layers of the network, determining the contribution of each node to its parent nodes. This process is described in Figure 2.

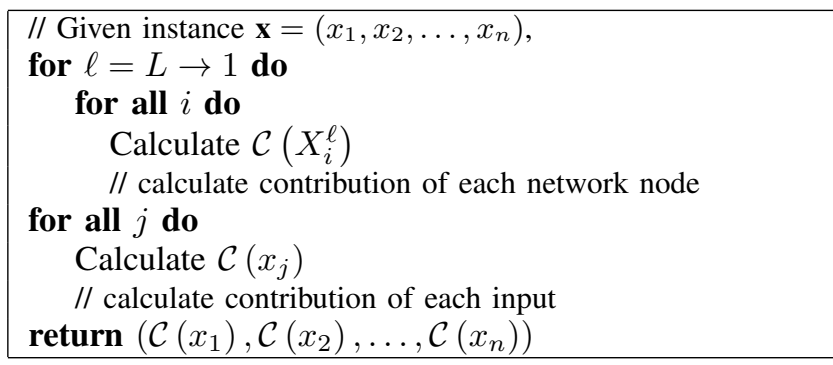

Fig. 2. The contribution-propagation algorithm. $\mathcal{C}\left(X_{i}^{\ell}\right)$ is the total contribution of node $X_{i}^{\ell}$. Recall that $\mathbf{x}=\left(x_{1}, \ldots, x_{n}\right)$ is the input to the network, thus $\mathcal{C}\left(x_{j}\right)$ is the total contribution of input $x_{j}$.

In order to complete the description of contribution propagation, we need only derive the equations for $\mathcal{C}\left(X_{k}^{L}\right)$ (the contribution of the network's output nodes) and $\mathcal{C}\left(X_{i}^{\ell} \mid X_{j}^{\ell+1}\right)$ (the partial contribution of internal nodes to their parents).

We have previously stated that $\mathcal{C}\left(X_{i}^{\ell} \mid X_{j}^{\ell+1}\right)$ represents how important $X_{i}^{\ell}$ was to $X_{j}^{\ell+1}$. To make this idea more concrete and well-founded, we impose the following constraints:

$$
\begin{aligned}
& \sum_{X_{i}^{\ell} \in \operatorname{ch}\left(X_{j}^{\ell+1}\right)} \mathcal{C}\left(X_{i}^{\ell} \mid X_{j}^{\ell+1}\right)=1 \\
& \text { and } \quad 0 \leq \mathcal{C}\left(X_{i}^{\ell} \mid X_{j}^{\ell+1}\right) \leq 1 .
\end{aligned}
$$


In other words, we require that $\mathcal{C}\left(X_{i}^{\ell} \mid X_{j}^{\ell+1}\right)$ be a distribution over $c h\left(X_{j}^{\ell+1}\right)$. The meaning of this distribution is the fraction of the value $X_{j}^{\ell+1}$ that is due to $X_{i}^{\ell}$.

With this in mind, we can see that each individual summand from Equation $1, \mathcal{C}\left(X_{i}^{\ell} \mid X_{j}^{\ell+1}\right) \mathcal{C}\left(X_{j}^{\ell+1}\right)$, is the portion of the contribution of $X_{j}^{\ell+1}$ that is due to $X_{i}^{\ell}$. Thus Equation 1 in its entirety tells us that a node's contribution $\mathcal{C}\left(X_{i}^{\ell}\right)$ is equal to all of the contributions in the parent's layer for which $X_{i}^{\ell}$ is responsible.

We now derive $\mathcal{C}\left(X_{i}^{\ell} \mid X_{j}^{\ell+1}\right)$ for $\mathrm{RBF}$ and maximum functions (the two types of nodes in our network). We will conclude with the definition of $\mathcal{C}\left(X_{k}^{L}\right)$, the contribution of the network outputs. This will complete our derivation of the contribution-propagation algorithm.

\section{B. Contribution to Radial Basis Function}

Consider a node $X_{j}^{\ell+1}$ in an $S$-layer. For convenience, let $\mathbf{X}=\operatorname{ch}\left(X_{j}^{\ell+1}\right)$ and $\mathbf{P}=\mathbf{P}_{j}^{\ell+1}$. Then the function computed by $X_{j}^{\ell+1}$ is the RBF

$$
X_{j}^{\ell+1}=\exp \left(-\beta\|\mathbf{X}-\mathbf{P}\|^{2}\right) .
$$

Our goal is to define a function $\mathcal{C}_{\mathrm{RBF}}\left(X_{i}^{\ell} \mid X_{j}^{\ell+1}\right)$ which is a distribution over $\mathrm{ch}\left(X_{j}^{\ell+1}\right)$ and which accurately describes the degree to which $X_{i}^{\ell}$ was responsible for the value of $X_{j}^{\ell+1}$.

Equation 4 is a measure of distance between the vectors $\mathbf{X}$ and $\mathbf{P}$. A closer distance yields a larger RBF value, and a further distance yields a smaller value. Thus we want $\mathcal{C}_{\mathrm{RBF}}\left(X_{i}^{\ell} \mid X_{j}^{\ell+1}\right)$ to be higher if $X_{i}^{\ell}$ is closer to $P_{i}$, meaning when $\left(X_{i}^{\ell}-P_{i}\right)^{2}$ is smaller. Moreover, the distance calculated by the RBF is tuned by the function $s(x)=\exp (-\beta x)$. Thus, we define $\mathcal{C}_{\mathrm{RBF}}\left(X_{i}^{\ell} \mid X_{j}^{\ell+1}\right)$ as:

$$
\mathcal{C}_{\mathrm{RBF}}\left(X_{i}^{\ell} \mid X_{j}^{\ell+1}\right) \stackrel{\text { def }}{=} \frac{\exp \left(-\beta\left(X_{i}^{\ell}-P_{i}\right)^{2}\right)}{Z},
$$

where $Z$ is a normalization term.

Recalling the constraints in Equations 2 and 3, we note that the constraint of Equation 3 implies that $Z>\exp \left(-\beta\left(X_{i}^{\ell}-\right.\right.$ $\left.P_{i}\right)^{2}$ ). To make $\mathcal{C}_{\mathrm{RBF}}\left(X_{i}^{\ell} \mid X_{j}^{\ell+1}\right)$ satisfy the constraint in Equation 2 , it must be normalized so that $\sum_{i} \mathcal{C}_{\mathrm{RBF}}\left(X_{i}^{\ell} \mid X_{j}^{\ell+1}\right)=1$. Thus we simply set the denominator $Z$ in Equation 5 to equal the sum over all children of the RBF, and we arrive at the full definition:

$$
\mathcal{C}_{\mathrm{RBF}}\left(X_{i}^{\ell} \mid X_{j}^{\ell+1}\right) \stackrel{\text { def }}{=} \frac{\exp \left(-\beta\left(X_{i}^{\ell}-P_{i}\right)^{2}\right)}{\sum_{X_{k}^{\ell} \in \operatorname{ch}\left(X_{j}^{\ell+1}\right)} \exp \left(-\beta\left(X_{k}^{\ell}-P_{k}\right)^{2}\right)} .
$$

Equation 6 agrees with our intuition: those children $X_{i}$ that are close to their target $P_{i}$ have a higher contribution.

\section{Contribution to Maximum Function}

A node $X_{j}^{\ell+1}$ in a $C$-layer calculates the maximum of its children,

$$
X_{j}^{\ell+1}=\max _{X_{i}^{\ell} \in \operatorname{ch}\left(X_{j}^{\ell+1}\right)} X_{i}^{\ell}
$$

As before, our goal is to define the function $\mathcal{C}_{\mathrm{MAX}}\left(X_{i}^{\ell} \mid X_{j}^{\ell+1}\right)$ which is a distribution over $X_{i}^{\ell}$ and which accurately describes the degree to which $X_{i}^{\ell}$ was responsible for the value of $X_{j}^{\ell+1}$.

To this end, we note that an input to a max function was important to the function if that input was itself the maximum value. Thus we view the max function as a type of switch, and we define

$$
\mathcal{C}_{\mathrm{MAX}}\left(X_{i}^{\ell} \mid X_{j}^{\ell+1}\right) \stackrel{\text { def }}{=} \begin{cases}1 / r & \text { if } X_{i}^{\ell} \in \mathcal{M} \\ 0 & \text { o.w. }\end{cases}
$$

where $\mathcal{M}=\left\{X_{i}^{\ell} \in \operatorname{ch}\left(X_{j}^{\ell+1}\right): X_{i}^{\ell}=X_{j}^{\ell+1}\right\}$, and $\mathcal{M}$ contains $r$ elements. That is, we divide the contribution equally among those inputs that shared the maximum value; those children who were not the maximum did not contribute.

\section{Contribution to an Additive Classifier}

Given a feature vector $\mathbf{X}^{L}$ classified with an additive classifier as $\hat{y}\left(\mathbf{X}^{L}\right)=\operatorname{sgn}\left[\sum_{i} f_{i}\left(X_{i}^{L}\right)+b\right]$, we define the contribution of feature $X_{i}^{L}$ to be $f_{i}\left(X_{i}^{L}\right)$ as in Poulin et al. [4]. We denote the feature's contribution by writing

$$
\mathcal{C}\left(X_{i}^{L}\right) \stackrel{\text { def }}{=} f_{i}\left(X_{i}^{L}\right) \text {. }
$$

Here we assume the additive classifier is a linear SVM [11]. Given the set $\mathcal{V}$ of support vectors, a linear SVM calculates $\hat{y}\left(\mathbf{X}^{L}\right)=\operatorname{sgn}\left[\sum_{\mathbf{V} \in \mathcal{V}} \alpha_{\mathbf{v}}\left\langle\mathbf{V}, \mathbf{X}^{L}\right\rangle+b\right]=$ $\operatorname{sgn}\left[\sum_{i} X_{i}^{L}\left(\sum_{\mathbf{V} \in \mathcal{V}} \alpha_{\mathbf{v}} V_{i}\right)+b\right]$, where $\langle\cdot, \cdot\rangle$ denotes the dot product. In this case, Equation 9 becomes

$$
\mathcal{C}_{\mathrm{SVM}}\left(X_{i}^{L}\right) \stackrel{\text { def }}{=} X_{i}^{L}\left(\sum_{\mathbf{V} \in \mathcal{V}} \alpha_{\mathbf{v}} V_{i}\right) .
$$

That is, Equation 10 gives the contribution of node $X_{i}^{L}$ to the SVM's classification of feature vector $\mathbf{X}^{L}$. A positive value gives the amount to which node $X_{i}^{L}$ contributes to a positive classification (or away from a negative classification); similarly a negative value gives the degree of $X_{i}^{L}$ 's contribution towards a negative (or away from a positive) classification.

\section{HiERARCHICAL NETWORK IMPLEMENTATION}

We implement a four-layer network (Figure 1), based on the network of Serre et al. [3]. The input image is preprocessed to form a $256 \times 256$ gray-scale image with local contrast enhancement. An S1 kernel is an 11×11-pixel Gabor filter. Using Equation 4, we apply a battery of Gabors at 8 orientations, 2 phases, and 4 scales, with $\beta=1.0$ for all S1 nodes. For each Gabor configuration, we subsample by centering an S1 node at every other pixel, resulting in a set of $64 \mathrm{~S} 1$ output maps, each of size $128 \times 128$. A C1 node pools over the two phases and a $5 \times 5$ spatial neighborhood of S1 outputs. We again subsample in the same way, resulting in $32 \mathrm{C} 1$ output maps, each of size $64 \times 64$. For an $\mathrm{S} 2$ node, the input is a $7 \times 7$ neighborhood of $\mathrm{C} 1$ nodes at all orientations, but at a single scale. The input vector and the kernel of each S2 node are each scaled to unit length $(\|\mathbf{X}\|=\|\mathbf{P}\|=1)$. We set $\beta=5.0$ for every $\mathrm{S} 2$ node. For each kernel, there is a corresponding S2 node centered at every other $\mathrm{C} 1$ node, resulting in multiple $32 \times 32 \mathrm{~S} 2$ output maps, one for each kernel and scale. Finally, a C2 node applies a $\max$ operation to all locations and all scales of a single kernel's 
$\mathrm{S} 2$ map. Thus the output of the $\mathrm{C} 2$ layer is a vector with one component per S2 kernel. This feature vector is passed to the linear SVM. We use the SVM ${ }^{\text {light }}$ package [11] with an unbiased SVM $(b=0)$. This allows a simpler derivation of our method without impacting the accuracy of the network.

\section{RESUlts}

We apply our contribution-propagation method in order to explain the classifications of hierarchical networks that are trained on different tasks. The first experiment is intentionally simple and controlled, and demonstrates that our approach accurately explains the network's classifications. The second experiment, which uses a well-known, real-world data set, shows how contribution propagation gives new insight into the network's performance.

\section{A. Simple Shapes}

In our first experiment, we use a simple artificial visual classification task to verify that our method's explanations are correct and understandable. Each training image contains a simple shape, either an 'L' shape (Figure 3B, positive class) or an inverted ' $\mathrm{L}$ ' shape (Figure $3 \mathrm{C}$, negative class). Noise is added by rotating the shape uniformly randomly within \pm 5 degrees and translating the shape to a random location, and $1 / f$ noise is added to the background. The noise ensures that the learned classifier is nontrivial.

Figure 3A shows the two learned S2 kernels around the vertex of the ' $L$ ' and inverted ' $L$ ' shapes. We input 20 training images (10 positive and 10 negative) to the network, and use the resulting feature vectors to train the SVM.

Our test images contain 9 possible shapes (Fig. 3 D), including both an ' $\mathrm{L}$ ' and inverted ' $\mathrm{L}$ ', each placed at a random position in a $3 \times 3$ grid and rotated randomly within \pm 5 degrees. Again, $1 / f$ noise is added to the background. Note that because both the positive and negative objects are present in the test image, we do not expect one classification over the other. The test images were designed to illustrate the authenticity of the contribution-propagation algorithm rather than to test the classification accuracy of the hierarchical network (accuracy will be addressed in the next section). All test images in this toy example were very near the decision boundary, which is reasonable given that both the positive and negative classes are present in each test image.

Using contribution propagation, we explain a test image's classification using false color as follows. First, we trace down through the layers of the network (Fig. 3E-H) using the algorithm presented in Figure 2. Thus Figure 2E shows the contribution of each node in the $S 2$ layer, Figure $2 \mathrm{~F}$ shows the contribution of each node in the $C 1$ layer, and so on. This results in the calculation of the contribution $\mathcal{C}\left(x_{i}\right)$ of each pixel $x_{i}$ (Fig. 3H). Pixels drawn in red contributed to a positive classification (' $\mathrm{L}$ '); pixels drawn in blue contributed to a negative classification (inverted ' $L$ '); pixels that did not contribute to the classification are drawn in green.

We see in this visualization (Figure $3 \mathrm{H}$ ) that our contribution-propagation algorithm correctly colored the pixels surrounding the area matching the learned kernels (red around the 'L' shape, and blue around the inverted ' $L$ ' shape). Our algorithm thus explains the classification of "undecided": there was a nearly equal "pull" between the pixels surrounding the ' $L$ ' (toward positive classification) and those surrounding the inverted ' $L$ ' (toward negative classification). This pixel-level explanation of how the image is interpreted by the network and classifier was provided automatically by our contributionpropagation algorithm, and gives evidence for the correctness of our algorithm.

\section{B. Real-World Images}

Next, we use the Caltech101 data set [12] to train the network and a linear, unbiased SVM in a binary classification task using categories of "chair" (positive class, corresponding to red in the visualizations) and "dalmatian" (negative class, corresponding to blue). The categories contain 60 images each. Using 10 splits for cross validation, we randomly choose 30 training images and 30 test images from each category. Following Serre et al. [3], the network "imprints" 1000 S2 kernels randomly from the S2 inputs of the training set, and the SVM is trained on the resulting network's output for each training image. Test images are classified with an average accuracy of $94 \%$, with a $3 \%$ standard deviation (a biased SVM achieved $93 \%$ accuracy with $1.2 \%$ standard deviation).

In Figure 4, we see that some images $(\mathrm{A}, \mathrm{C})$ are correctly classified primarily due to the pixels of the object itself $(B, D)$. However, our method also reveals some surprising behavior of the network and classifier (F, H): it appears that some images were correctly classified due to features extracted primarily from the image's background. In Figure 4F, this may be less surprising as the background is quite similar to the dalmatian. However, in Figure $4 \mathrm{H}$, it is unclear why the background (dark red) was taken as evidence for the presence of a chair (or, possibly, absence of a dalmatian). Such an unexpected explanation offered by the contribution-propagation method can be useful to the user who is trying to create a system that will generalize well; the user can see that, at least in some cases, the network is basing its classification on features that are not relevant to the general task, due to either deficiencies in the network or spurious correlations in the data set.

A natural question is how often a correct classification is "surprising" (that is to say, a correctly classified image where the background appears to contribute more than the object). Formulating a metric to define such a surprising classification is beyond the scope of the present work. However, a subjective visual inspection of the classified images reveals 5 of the 60 classifications of test images to be of this nature.

As a final test of the classification explanations provided by the contribution-propagation algorithm, we edit an image to include both a dalmatian and a chair (Figure 4I). This image was classified by the network as negative (dalmatian), and the contribution propagation algorithm explains this classification. Figure $4(\mathrm{~J})$ shows that, although there were features associated with the chair class on the right side of the image (yellow, light red), the features extracted from the pixels belonging to the dalmatian were weighted more heavily (deep blue).

Some readers may feel that the small number of training images used may cast doubt on the validity of the trained classifier. However, note that researchers often benchmark their computer-vision system by measuring its performance on the 


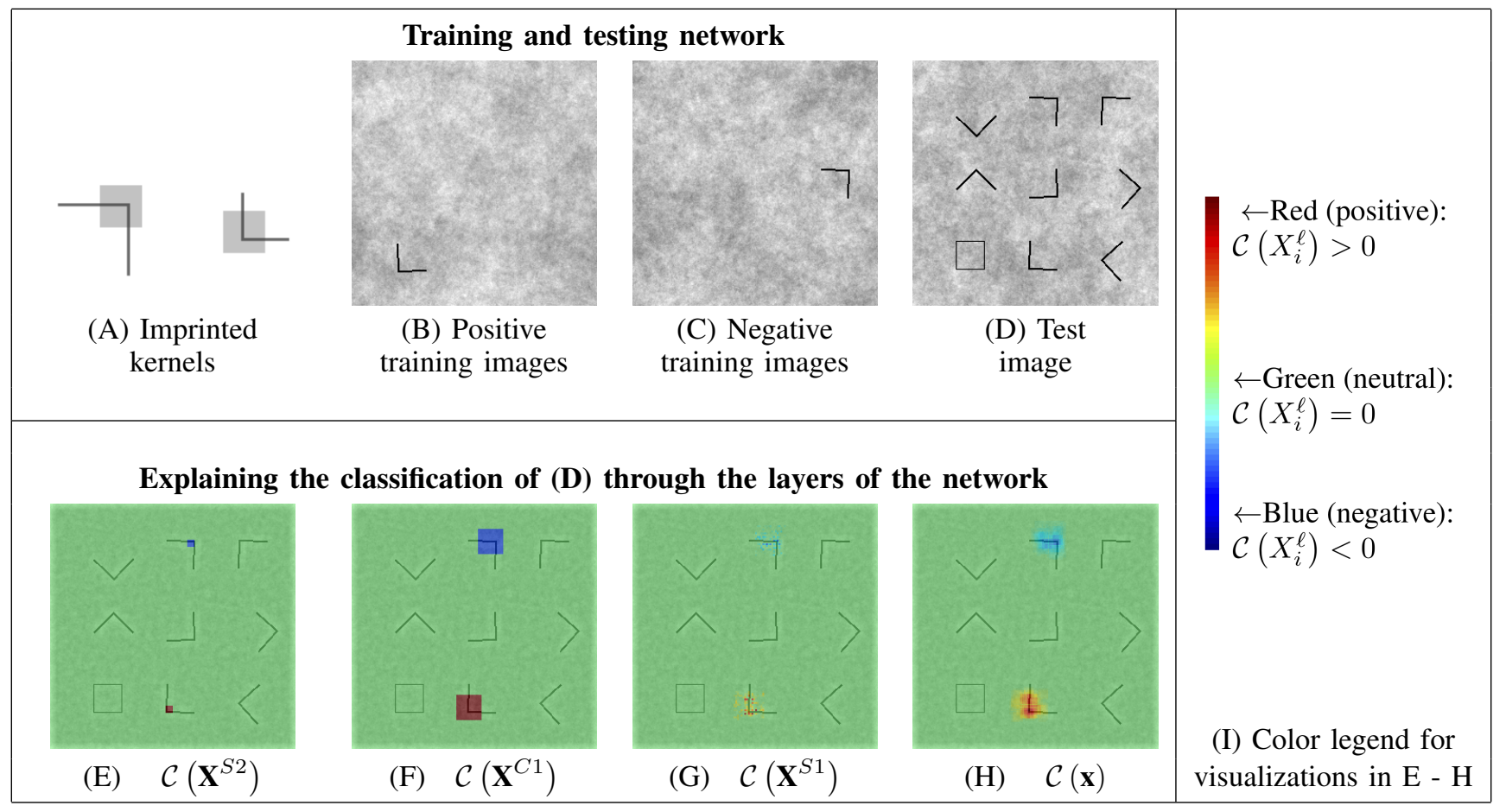

Fig. 3. (Figure best viewed in color.) Visualizing the binary classification of images containing simple shapes. The contribution of the nodes in each layer verifies the correctness of our algorithm. Two S2 kernels are used (shaded squares, A). A linear SVM is trained on images containing either an 'L' shape (B, positive class) or an inverted ' $\mathrm{L}$ ' (C, negative class). We present a test image (D), and use contribution propagation to visualize the contribution of all layers $(\mathrm{E}-\mathrm{H})$. Note that the image is drawn in the background of $(\mathrm{E}-\mathrm{H})$ in order to better explain the contribution of each region. Colors correspond to the pixel's contribution, as shown in the legend (I). These visualizations give evidence for the correctness of our contribution propagation algorithm.

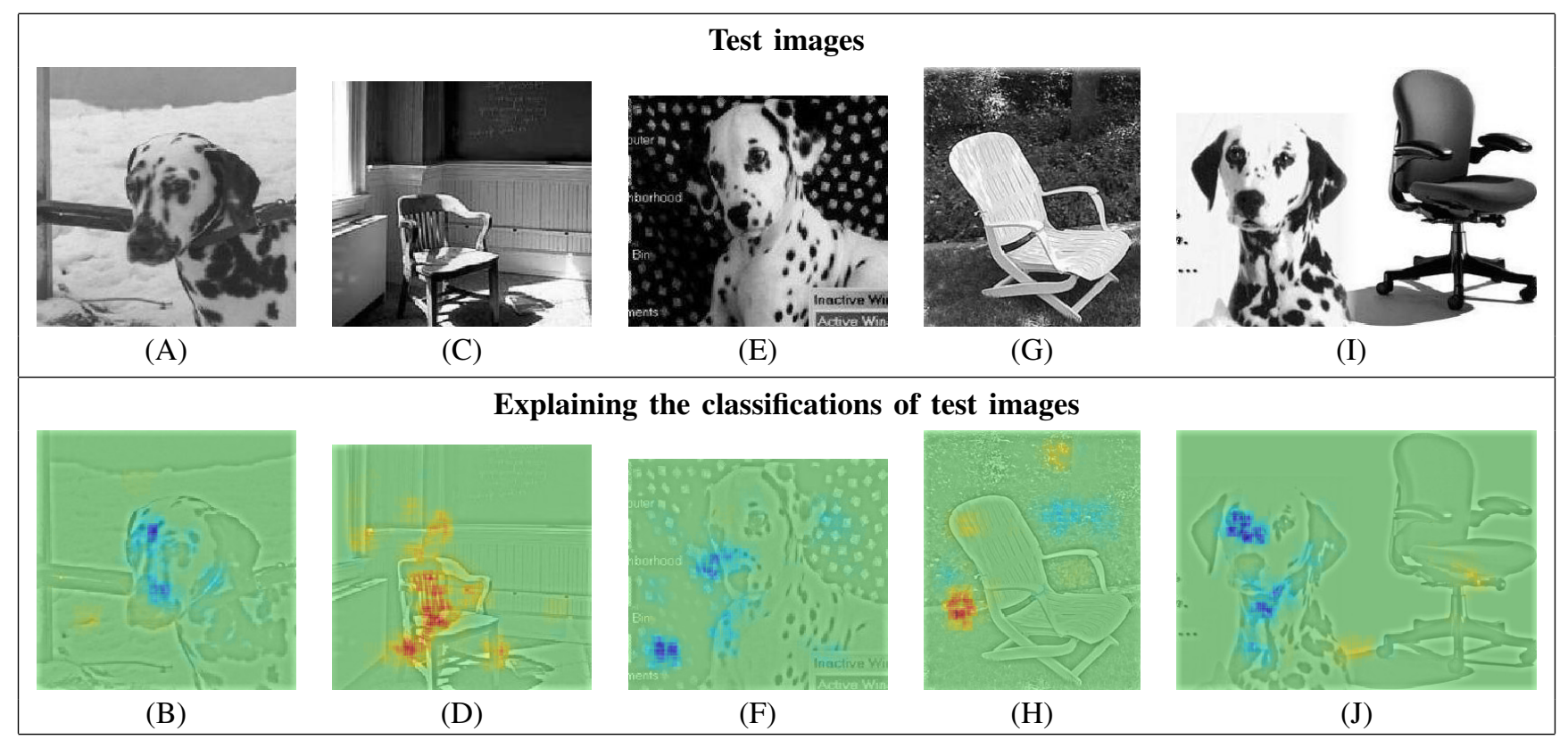

Fig. 4. (Figure best viewed in color.) Visualizations from the classification of chairs (positive) vs. dalmatians (negative), from the Caltech101 database. Colors correspond to the legend in Figure 3 (I): positive contribution (toward chair) is denoted with red, and negative contribution (toward dalmatian) with blue. Some images (A, C) are correctly classified because of the contribution of pixels that belong to the object being classified (B, blue on dalmatian; D, red on chair). Other images that contain confusing patterns in the background $(\mathrm{E}, \mathrm{G})$ are still correctly classified, but partially due to the contribution of background pixels (F, blue on background; $\mathrm{H}$, red on background). An image manipulated to contain both objects (I) is classified as dalmatian, and this classification is intuitively explained by the contribution-propagation algorithm (J). 
Caltech101 dataset using 30 images per class as training data [13]. Thus our experiment was designed to mimic a benchmarking process familiar to many computer-vision researchers. In this light, the surprising results presented in Figure 4 hint at an important question to the computer-vision community: does high performance on this dataset indicate a system's capacity for object recognition, or merely for learning spurious statistical (background) cues? The prevalence of this dataset makes this question all the more pressing.

\section{CONCLUSIONS}

We presented a novel method for explaining the classifications of hierarchical networks with additive classifiers, having reviewed why traditional approaches such as a sensitivity analysis fail to give satisfactory explanations. Our method extends the contribution-based explanations of Poulin et al. [4], and determines the contribution of each input based on the internal calculations of the network.

We empirically validate our method's explanations with a simple object-recognition task using artificial data. We apply our method to a binary classification task using a well-known set of natural images, revealing surprising artifacts in the way that some images are classified. In particular, we see that some images are correctly classified because of the contribution of pixels belonging to the image's background (Figure $4 \mathrm{~F}, \mathrm{H}$ ). This behavior is surprising when the task is completed with high accuracy, but it is also very useful to the user of the machine-learning algorithm. Such information provided by our method can help the user to tune the algorithm for better generalizability, as well as to create data sets without artifacts in the background.

In order to visualize our method's explanations, we classify with an unbiased SVM. Although the lack of bias may, in some cases, lead to lower classification accuracy, it did not for any task we implemented. We stress that the lack of bias was chosen merely for visualization, and that the contributionpropagation method can be implemented with biased functions as well.

In future work, the contribution-propagation method may also be applied to different types of hierarchical networks, for example those using convolutions or different subsampling methods.

\section{ACKNOWLEDGMENTS}

This material is based upon work supported by the National Science Foundation under Grant Nos. 1018967 and 0749348, as well as the Laboratory Directed Research and Development program at Los Alamos National Laboratory (Project 20090006DR). Any opinions, findings, conclusions or recommendations expressed in this material are those of the authors and do not necessarily reflect the views of the funding agencies.

\section{REFERENCES}

[1] R. Rifkin, J. Bouvrie, K. Schutte, S. Chikkerur, M. Kouh, T. Ezzat, and T. Poggio, "Phonetic classification using hierarchical, feed-forward, spectro-temporal patch-based architectures," Massachusetts Institute of Technology, Tech. Rep. MIT-CSAIL-TR-2007-019, 2007.
[2] Y. LeCun, L. Bottou, Y. Bengio, and P. Haffner, "Gradient-based learning applied to document recognition," Proceedings of the IEEE, vol. 86 , no. 11,1998 .

[3] T. Serre, A. Oliva, and T. Poggio, "A feedforward architecture accounts for rapid categorization," Proceedings of the National Academy of Sciences, vol. 104, no. 15, 2007.

[4] B. Poulin, R. Eisner, D. Szafron, P. Lu, R. Greiner, D. Wishart, A. Fyshe, B. Pearcy, C. MAcDonell, and J. Anvik, "Visual explanation of evidence in additive classifiers," in Proceedings of 18th Conference on Innovative Applications of Artificial Intelligence. IAAI, July 2006.

[5] L. Fu, "Rule generation from neural networks," IEEE Transactions on Systems, Man and Cybernetics, vol. 24, no. 8, 1994.

[6] T. Masquelier and S. Thorpe, "Unsupervised learning of visual features through spike timing dependent plasticity," PLoS Comp. Bio., vol. 3, no. 2, 2007.

[7] E. Strumbelj and I. Kononenko, "An efficient explanation of individual classifications using game theory," Journal of Machine Learning Research, vol. 11, no. 1, 2010.

[8] D. Baehrens, T. Schroeter, S. Harmeling, M. Kawanabe, K. Hansen, and K. Müller, "How to explain individual classification decisions," Journal of Machine Learning Research, vol. 11, pp. 1803-1831, 2010.

[9] M. Riesenhuber and T. Poggio, "Hierarchical models of object recognition in cortex," Nature Neuroscience, vol. 2, no. 11, 1999.

[10] M. Dredze, K. Crammer, and F. Pereira, "Confidence-weighted linear classification," in International Conference on Machine Learning, 2008.

[11] T. Joachims, "Making large-scale SVM learning practical." in Advances in Kernel Methods - Support Vector Learning, B. Schölkopf, C. Burges, and A. Smola, Eds. MIT Press, 1999.

[12] L. Fei-Fei, R. Fergus, and P. Perona, "Learning generative visual models from few training examples: an incremental Bayesian approach tested on 101 object categories," in IEEE. CVPR 2004, Workshop on Generative-Model Based Vision, 2004.

[13] A. Bosch, A. Zisserman, and X. Munoz, "Representing shape with a spatial pyramid kernel," in Proceedings of the 6th ACM international conference on Image and video retrieval, 2007. 